\title{
Antibody-free multiplex measurement of 23 human cytokines in primary cell culture secretome using targeted mass spectrometry
}

\author{
Annika Krueger ${ }^{1}$, Thomas Stoll ${ }^{2}$, Alok K. Shah ${ }^{2}$, Rohit Sinha ${ }^{1}$, Ian H. Frazer ${ }^{1}$, and Michelle \\ M. Hill ${ }^{1,2 *}$
}

${ }^{1}$ The University of Queensland Diamantina Institute, Faculty of Medicine, The University of Queensland, Woolloongabba, Brisbane QLD 4102, Australia

${ }^{2}$ QIMR Berghofer Medical Research Institute, Brisbane QLD 4006, Australia

Corresponding Author

*Email: Michelle.Hill@ qimrberghofer.edu.au

\begin{abstract}
Cytokines are commonly measured by immunoassays however these have limited multiplexing capacity, are costly and can exhibit cross-reactivity. Multiple reaction monitoring (MRM) mass spectrometry is a robust method to quantify analytes with high specificity and multiplexing ability, hence we aimed to investigate its suitability as an alternative cost-effective method for cytokine measurement. Human keratinocyte conditioned media spiked with recombinant cytokines was used as an experimental system to evaluate sensitivity, linearity and reproducibility of an MRM assay targeting 79 peptides representing 23 human cytokines. Our MRM method was able to identify 21 cytokines by two or more unique peptides, and two cytokines by a single unique peptide. In a serum-free matrix, the median LOD and LOQ for cytokine peptides was 130 and $433 \mathrm{pg} / \mathrm{mL}$, respectively. The presence of serum increased median LOD and LOQ by about 2.3-fold. The assay shows excellent replicate consistency with $8 \%$ intra- and $12 \%$ inter-day coefficient of variations. We found high $\mathrm{pH}$ reversed-phase fractionation a useful tool to increase assay sensitivity with the drawback of increasing its variability by approximately $10 \%$. Overall, our results suggest utility of a multiplex cytokine MRM for routine measurement of secreted cytokines in cellular experiments under low serum conditions. Additional enrichment steps will be required in high complexity matrices such as serum.
\end{abstract}

\section{SUPPORTING INFORMATION}

Supplemental Materials and Methods

Figure S1: Absolute number and percentage of cytokines with detectable peptides showing linearity

Figure S2: Examples of calibration curves and MS signal intensity of internal standard ovalbumin

Table S1: MRM transition list exported from Skyline (separate Excel file)

Table S2: $\mathrm{LOD} / \mathrm{LOQ} / \mathrm{R}^{2}$ calculations (separate Excel file)

Table S3: Reproducibility experiment: CV calculations (separate Excel file) 


\section{SUPPLEMENTAL MATERIALS AND METHODS}

\section{MRM/MS assay development}

Preparation of cytokine standards and ovalbumin quality control

Twenty-three human recombinant cytokine proteins (Table 1) were purchased from Biolegend and R\&D Systems. An aliquot of $500 \mathrm{ng}$ of each standard protein was diluted with milliQ water to a final volume of $100 \mu \mathrm{L}$ containing $1 \%(\mathrm{w} / \mathrm{v})$ sodium deoxycholate (SDC) and $100 \mathrm{mM}$ triethylammonium bicarbonate buffer (TEAB, $\mathrm{pH} 8.5)$. Pooled proteins were then heated for 5 minutes at $95^{\circ} \mathrm{C}$, followed by cooling on ice. Proteins were reduced with $2 \mu \mathrm{L}$ of $0.5 \mathrm{M}$ tris(2-carboxyethyl)phosphine (neutralised to $\mathrm{pH} 7$ with ammonium hydroxide, $10 \mathrm{mM}$ final concentration) for 30 minutes at $60^{\circ} \mathrm{C}$ and then alkylated with $9 \mu \mathrm{L}$ of $0.5 \mathrm{M}$ iodoacetamide (40 mM final concentration) in the dark at $37^{\circ} \mathrm{C}$ for 30 minutes. To co-precipitate proteins, $1 \mu \mathrm{L}$ of sequencing-grade modified trypsin (Promega Madison, WI; trypsin to protein ratio of 1:100) was added to the side of the Eppendorf tube and quickly flushed into the solution with $1.1 \mathrm{~mL}$ methanol $\left(100 \%\right.$, at $\left.-20^{\circ} \mathrm{C}\right)$. The standard mixture was immediately vortexed for $30 \mathrm{~s}$ and then incubated overnight at $-20^{\circ} \mathrm{C}$. The following day, protein precipitates were harvested by centrifugation at $15,000 \mathrm{xg}$ for 15 minutes $\left(4^{\circ} \mathrm{C}\right)$. Pellets were washed twice, once with $1 \mathrm{~mL}$ of $90 \%(\mathrm{v} / \mathrm{v})$ methanol at $-20^{\circ} \mathrm{C}$ and finally with $1 \mathrm{~mL}$ of $100 \%$ methanol at $-20^{\circ} \mathrm{C}$ and centrifuged each time as before. Precipitates were allowed to air dry and the dried pellet was re-suspended in $100 \mathrm{mM}$ TEAB buffer (pH 8.5) containing $5 \% \mathrm{ACN}$ by vortexing and sonicating ( 1 minute each). The standard mixture was incubated at $37^{\circ} \mathrm{C}$ for 2 hours. Then trypsin was added (trypsin:protein ratio $1: 100$ ) for overnight digestion at $37^{\circ} \mathrm{C}$. The next day, digests were acidified by adding 1:1 (v/v) $10 \%$ formic acid (FA) (= $250 \mathrm{ng} / \mu \mathrm{L}$ final protein concentration) and stored at $-80^{\circ} \mathrm{C}$ until analysis.

Sample preparation of quality control (QC) ovalbumin (A5503, Sigma) was carried out as described above for cytokine standards with the exception that $50 \mu \mathrm{g}$ ovalbumin was diluted in $2 \%$ sodium dodecyl sulfate (SDS) instead of $1 \%$ SDC.

\section{Shotgun proteomics and mass spectral library generation}

Tryptic digests of recombinant cytokine proteins were analysed on an EASY-nLC 1000 UHPLC system (Thermo Scientific, Bremen, Germany) coupled to a Q Exactive Plus mass spectrometer (Thermo Scientific) via an EASYSpray ion source (Thermo Scientific). Acidified digests were further diluted with $0.1 \%(\mathrm{v} / \mathrm{v}) \mathrm{FA}$ and centrifuged for 10 minutes at $15,000 \mathrm{xg}\left(4^{\circ} \mathrm{C}\right)$ prior to injection. For data-dependent acquisition analysis, $1 \mu \mathrm{L}$ ( $9 \mathrm{ng}$ of each 
standard) were loaded onto a nanoViper PepMap100 C18 trap column ( $3 \mu \mathrm{m}, 75 \mu \mathrm{m}$ x $20 \mathrm{~mm})$ at 800 bar and then separated on an EASY-Spray column (PepMap C18 $2 \mu \mathrm{m} 100 \AA, 75 \mu \mathrm{m}$ x $500 \mathrm{~mm}$ ) at a flow rate of $250 \mathrm{~nL} / \mathrm{min}$. Eluent A was $0.1 \% \mathrm{FA}$ in milliQ water and eluent B was $0.1 \% \mathrm{FA}$ in ACN. The sample was trapped at $100 \%$ eluent $\mathrm{A}$ and separated at $50^{\circ} \mathrm{C}$ using the following gradient: 0 min (3\% eluent $\left.\mathrm{B}\right)-1 \mathrm{~min}(7 \% \mathrm{~B})-41 \mathrm{~min}$ $(22 \%$ B $)-49 \min (40 \%$ B $)-50 \min (95 \%$ B $)-65 \min (95 \%$ B $)-66 \min (3 \%$ B). Column-separated peptides were electrosprayed into the mass spectrometer through the uncoated silica emitter integrated into the EASY-Spray column-emitter design with the following conditions: Spray voltage was $1.8 \mathrm{kV}$ with no sheath, sweep or auxiliary gases used. The heated capillary temperature was set to $250^{\circ} \mathrm{C}$ and the S-lens to $60 \%$.

The mass spectrometer was controlled using Xcalibur 3.0 software (Thermo Scientific) and was operated in positive ion and data-dependent acquisition mode to automatically switch between full scan MS and MS/MS acquisition. Full scan MS spectra (m/z 350-1400) were acquired with a resolving power set to 70,000 (at $200 \mathrm{~m} / \mathrm{z}$ ) after accumulation to a target value of $3 \times 10^{6}$ with a maximum injection time of $100 \mathrm{~ms}$. The top 20 most intense ions with charge states $\geq 2+$ were sequentially isolated and fragmented using higher-energy collisional dissociation with a normalized collision energy of 28. MS/MS spectra were acquired at a resolution of 17,500 with a target value of $5 \times 10^{5}$ and a maximum injection time of $55 \mathrm{~ms}$ using an isolation window of $1.4 \mathrm{Da}$. Underfill ratio was at $1 \%$ and dynamic exclusion was set to 20 s.

All MS/MS data were converted to Mascot generic format (mgf) files using Proteome Discoverer 2.1 (Thermo Scientific) and analysed using the Mascot search engine (Matrix Science, London, UK; version 2.4.1). Mascot server access was provided by the Walter and Eliza Hall Institute of Medical Research (Melbourne, Australia). SwissProt (taxonomy: Homo sapiens; 20,202 protein sequences; May 2016) was used as target database for searches alongside with a contaminant database (139 sequences) maintained by the Walter and Eliza Hall Institute of Medical Research. MS/MS data were searched assuming trypsin digestion with two missed cleavages. Precursor and fragment ion tolerance was set to $\pm 10 \mathrm{ppm}$ and $\pm 0.02 \mathrm{Da}$, respectively. Carbamidomethylation of cysteine was specified as a fixed modification. Acetylation of the protein $\mathrm{N}$-terminus, oxidation of methionine and deamidation of glutamine and asparagine were set as variable modifications. Cytokine standards spectral libraries were built in Skyline-daily ${ }^{8}$ (cut-off score: 0.99 and 0.95) using Mascot DAT file search results.

\section{Selection of cytokine peptides and transitions for MRM/MS assay}

Cytokine sequences were downloaded from UniProt Knowledgebase and imported into Skyline-daily. Peptide and transition selection was an iterative process. The first round of selection was based on cytokine spectral 
libraries and filter settings. Peptide filter settings were not overly stringent at first, i.e. methionine and ragged end containing peptides were accepted if these showed a very good ranking and intense fragment-rich spectra. Further peptide filter tab settings included: peptide length 5-25 amino acids, N-terminal amino acids not excluded. Transition filter tab settings were: precursor charge $2+$ and $3+$, ion charge $1+$ and $2+$, ion type y and $b$. The top five most intense product ions were selected from the library spectra that also matched the filter tab. Peptides with a very poor ranking and low-intensity fragment-poor spectra were deleted. The resulting transition list ( $~ 900$ transitions) was exported and targeted MRM data was acquired ( 180 transitions per run, dwell time $10 \mathrm{~ms})$ injecting the cytokine standard digest. Data acquired in the MRM runs were imported into Skyline and the transition list further refined, i.e. peptides and precursors with no or poor signal were deleted. For cytokines that had no, only one, or non-ideal peptides (e.g., non-unique, contain PTMs, ragged ends, and/or methionine residues), a second round of selection was performed by generating transitions in silico based on the above stated filter settings with slight adjustments (i.e. ion type y only, 4 product ions from $(\mathrm{m} / \mathrm{z}>$ precursor) -1 , product ions $\mathrm{N}$ terminal to proline included, precursor $\mathrm{m} / \mathrm{z}$ exclusion window of 5 ). Charge state $3+$ precursors with $\mathrm{m} / \mathrm{z}<300$ were deleted manually from the list. Lastly, ion selection was expanded by adding b- and more y- product ions to the list to find the best possible transitions for filter settings based peptides. MRM data was acquired using the adjusted method including in silico transitions and subsequently the transition list was refined again to eliminate poor responders.

In a separate experiment, the transition list for ovalbumin peptides was generated in silico with the following filter settings: peptide length 5-45 amino acids, N-terminal amino acids and ragged ends not excluded, methionine containing peptides excluded. Transition filter tab settings: precursor charge $2+$ and $3+$, ion charge $1+$ and $2+$, ion type y and b, 5 product ions from $(\mathrm{m} / \mathrm{z}>$ precursor) -1 , product ions $\mathrm{N}$-terminal to proline included, precursor $\mathrm{m} / \mathrm{z}$ exclusion window of 5 . The ovalbumin digest $(1 \mu \mathrm{g} / \mu \mathrm{L})$ was diluted 1:50 with $5 \%$ FA to obtain a $20 \mathrm{ng} / \mu \mathrm{L}$ solution and $20 \mathrm{ng}$ was loaded onto column for method development. MRM data was acquired and the original transition list amended by deleting peptides and transitions with poor performance.

The final MRM assay contained 371 transitions from 24 proteins (ovalbumin and 23 cytokine sequences) and 81 peptides with a retention time window of $\pm 3 \mathrm{~min}$ and a cycle time of $2 \mathrm{~s}$ (equals $\sim 12$ data points across peak) resulting in a minimum dwell time of $10.6 \mathrm{~ms}$. Supplemental Table S1 contains a detailed list of transitions. All cytokine and ovalbumin peptides that were included in this final MRM method were checked for uniqueness using 
the Skyline background proteome function (human proteome downloaded from Uniprot: human AND reviewed:yes AND organism:"Homo sapiens (Human) [9606]" AND proteome:up000005640). Further, the opensource tool Protter was used to inspect peptides for PTMs. ${ }^{13}$ Finally, SRM collider ${ }^{18}$ was used to investigate transition interferences in the given background proteome (here: Human (tryptic)). The results from each of these searches (Skyline background proteome function, Protter and SRM collider) are indicated in Table 1.

\section{Cell culture}

Primary human keratinocytes were isolated from actinic keratosis lesions collected at the Princess Alexandra Hospital Dermatology Centre (Brisbane, Australia) with patient consent and institutional approval. Keratinocytes were cultured in media composed of 70\% F-12, 25\% DMEM (both Gibco, USA), 5\% FCS (HyClone, GE healthcare, USA) and the following supplements: $100 \mathrm{U} / \mathrm{mL}$ penicillin / streptomycin / glutamine and $10 \mathrm{ng} / \mathrm{mL}$ recombinant human epidermal growth factor (both Gibco), $8.4 \mathrm{ng} / \mathrm{mL}$ cholera toxin, $5 \mu \mathrm{g} / \mathrm{mL}$ insulin, $24 \mu \mathrm{g} / \mathrm{mL}$ adenine and 1:1000 $10 \mu \mathrm{M}$ Rho Kinase inhibitor (all purchased from Sigma Aldrich). Viability of the cells was routinely assessed by trypan blue exclusion assay and was consistently higher than $90 \%$. Experiments were performed up to passage three in 6-well plates using 15,000 cells per well. Three days post seeding, culture media was removed and keratinocytes were washed 5x with PBS, and subsequently, $2 \mathrm{~mL}$ of either $0.1 \%$ FCS or serumfree media was added. After 24 hours, cell supernatants were collected, pooled and divided into aliquots (14 x $10 \mathrm{~mL}$ aliquots $-7 \mathrm{x}$ no serum; 7x $0.1 \%$ FCS). All aliquots were spiked with 23 recombinant cytokine proteins at seven different concentrations $\left(1000,500,250,125,75,37.5\right.$ and $0 \mathrm{pg} / \mathrm{mL}$ of each cytokine) and stored at $-80^{\circ} \mathrm{C}$ until further sample preparation.

\section{Sample preparation of conditioned media (CM)}

The spiked keratinocyte CM samples were concentrated using an Amicon Ultra-15 ultrafiltration device (Ultracel$10 \mathrm{kD}$ regenerated cellulose membrane, Merck Millipore). Filter units were rinsed with $10 \mathrm{~mL}$ milliQ water before usage. $\mathrm{CM}$ aliquots were centrifuged for 10 minutes at $3,100 \mathrm{xg}\left(4^{\circ} \mathrm{C}\right)$ and supernatants were loaded into the Amicon device. The filter units were then centrifuged for $15 \mathrm{~min}$ at $3,100 \mathrm{xg}\left(4^{\circ} \mathrm{C}\right.$; Eppendorf centrifuge $5810 \mathrm{R}$ with swing bucket rotor \#A-4-81) followed by three washes (with $10 \mathrm{~mL}$ of PBS buffer; spun at $3,100 \times \mathrm{xg}$ at $4^{\circ} \mathrm{C}$ for $15 \mathrm{~min}$, except last wash step $60 \mathrm{~min}$ spin). Lastly, the retentate $(\approx 140-200 \mu \mathrm{L})$ was recovered and all samples were adjusted to a volume of $200 \mu \mathrm{L}$ with PBS, if required. The protein content in concentrated CM samples was 
assessed via NanoDrop technology (Thermo Scientific) at A280. Additionally, a Pierce BCA protein assay was performed according to the provided 'Microplate procedure' protocol.

An aliquot of $1.6 \mathrm{ng}$ of ovalbumin (per $1 \mu \mathrm{g}$ of sample) was spiked into all samples as QC. Each sample of concentrated CM $0.1 \%$ FCS $(46.5 \mu \mathrm{L}$, equals $\approx 100 \mu \mathrm{g}$ total protein) was diluted with $10 \%$ SDS (4\% final conc.) and $1 \mathrm{M}$ TEAB ( $\mathrm{pH} 8.5 ; 100 \mathrm{mM}$ final concentration) to a final sample volume of $100 \mu \mathrm{L}$, followed by heat treatment $\left(5\right.$ minutes at $\left.95^{\circ} \mathrm{C}\right)$ and cooling on ice. Concentrated $\mathrm{CM}$ samples without FCS had a 5-6x lower protein content compared to $0.1 \%$ FCS samples, hence a larger sample volume was processed and thus the treatment slightly adjusted: $40 \mu \mathrm{L}$ of $10 \%$ (w/v) SDS (2\% final conc.) was added to $150-200 \mu \mathrm{L}$ of sample aliquots (=60$80 \mu \mathrm{g}$ protein) followed by heating and cooling steps as above. The volume of all samples were reduced to about $60-70 \mu \mathrm{L}$ by vacuum centrifugation $\left(60\right.$ minutes at $\left.45^{\circ} \mathrm{C}\right)$. TEAB ( $\mathrm{pH} 8.5 ; 100 \mathrm{mM}$ final concentration) was added and volume adjusted with milliQ water to a final sample volume of $100 \mu \mathrm{L}$. Reduction, alkylation and tryptic digestion were performed as described above with minor adjustments: Dried protein pellets were re-suspended in $50 \mathrm{mM}$ TEAB buffer ( $\mathrm{pH} 8.5$ ) containing $5 \%$ ACN to obtain a $1 \mu \mathrm{g} / \mu \mathrm{L}$ protein concentration. Tryptic digests were stored at $-80^{\circ} \mathrm{C}$ without prior acidification until further analysis. $\mathrm{CM}$ cytokine digests were either fractionated as described below or directly analysed via MS.

\section{High pH (HpH) reversed-phase peptide fractionation}

Peptide fractionation was performed according to a method published by Keshishian et al. ${ }^{14}$ with modifications. Briefly, an aliquot of $50 \mu \mathrm{g}$ protein digest was acidified to $1 \% \mathrm{FA}$ and dried for 45 minutes in a vacuum concentrator at $45^{\circ} \mathrm{C}$. Digests were reconstituted in $105 \mu \mathrm{L}$ of $10 \mathrm{mM}$ ammonium formate $/ 2 \% \mathrm{ACN}(\mathrm{pH} 10)$ and $100 \mu \mathrm{L}$ were loaded onto a Zorbax 300 Extend-C18 column $(3.5 \mu \mathrm{m}, 300 \AA$ A, 2.1x150 mm; Agilent, Santa Clara, USA) connected to a Zorbax 300 Extend-C18 guard column $(5 \mu \mathrm{m}$ 2.1x12.5 mm). Peptides were fractionated on an Agilent 1200 series HPLC instrument by $\mathrm{HpH}$ reversed-phase chromatography comprising the following mobile phases and gradient: Eluent A was $10 \mathrm{mM}$ ammonium formate (pH 10) in milliQ water and eluent $\mathrm{B}$ was $10 \mathrm{mM}$ ammonium formate $(\mathrm{pH} \mathrm{10)}$ in $90 \% \mathrm{ACN}$. Total method runtime was 85 minutes with the following gradient: $0 \min (0 \%$ eluent $\mathrm{B})-5 \min (0 \% \mathrm{~B})-13 \min (12.5 \% \mathrm{~B})-46 \min (28.5 \% \mathrm{~B})-51 \min (34 \% \mathrm{~B})-62.5 \min$ $(60 \%$ B $)-63 \min (100 \%$ B $)-68.5 \min (100 \%$ B $)-69 \min (0 \%$ B $)-85 \min (0 \%$ B $)$. A flow rate of $0.2 \mathrm{~mL} / \mathrm{min}$ and column temperature of $25^{\circ} \mathrm{C}$ were maintained. 96 fractions per sample were collected in 0.6 min slices throughout the entire peptide elution from $7.5 \mathrm{~min}$ to $65.1 \mathrm{~min}$ into a 96 -well microplate (Greiner, PP, V-bottom, 
\#651201) which was monitored by a multi wavelength detector at 215, 220, and $280 \mathrm{~nm}$. All 96 fractions were recombined into eight fractions by using a concatenation strategy of pooling early, mid and late eluting fractions. Pooled fractions $(\sim 1.1 \mathrm{~mL})$ were acidified to $1 \% \mathrm{FA}$ and dried for a total of 5 hours at $45^{\circ} \mathrm{C}$ constant heating, followed by storage at $-80^{\circ} \mathrm{C}$ until LC/MS analysis.

\section{Nano LC-MRM/MS analysis}

For LC-MRM/MS analysis, dried $\mathrm{HpH}$-fractionated samples were re-suspended in 5\% FA containing $3 \% \mathrm{ACN}$ to obtain a $0.25 \mu \mathrm{g} / \mu \mathrm{L}$ peptide concentration, centrifuged for 5 minutes at $15,000 \times \mathrm{xg}\left(4^{\circ} \mathrm{C}\right)$ and $4 \mu \mathrm{L}$ (equivalent to $1 \mu \mathrm{g}$ ) loaded onto the column. Non-fractionated samples ( $\mathrm{pH} 8.5$ ) were acidified to $5 \%$ FA containing $2.5 \%$ ACN by diluting samples $1: 1(\mathrm{v} / \mathrm{v})$ with $10 \%$ FA $(=0.5 \mu \mathrm{g} / \mu \mathrm{L}$ peptide concentration), centrifuged and $2 \mu \mathrm{L}$ (equivalent to $1 \mu \mathrm{g}$ ) injected onto column. The acidified cytokine standard digest in clean background was run with every experiment as reference (column loading: 2 ng per protein).

Targeted MS analysis was performed on a 1260 Infinity nanoflow LC coupled to a 6490 iFunnel triple quadrupole mass spectrometer via Chip Cube nanospray interface (Agilent Technologies, Santa Clara, CA). Peptide separation was performed on microfluidic HPLC chip Polaris-HR-Chip3C18 (II) (pn G4240-62030, Agilent) with a $360 \mathrm{~nL}$ enrichment column and a $150 \mathrm{~mm}$ x $75 \mu \mathrm{m}$ analytical column, both packed with Polaris C18-A (3 $\mu \mathrm{m}$, $180 \AA$ A) stationary phase. The autosampler temperature was set to $4^{\circ} \mathrm{C}$. Chromatographic separation comprised the following conditions: Eluent A was milliQ water and eluent B was ACN with both eluents containing $0.1 \%$ FA. A flow rate of $2 \mu \mathrm{L} / \mathrm{min}$ was used for sample loading with $3 \%$ eluent B via capillary pump and $300 \mathrm{~nL} / \mathrm{min}$ was applied for peptide separation via nanoflow pump. A total method runtime of 45 min with a 32 min gradient was employed for peptide separation: $0 \min (4 \%$ B) - $32 \min (33 \%$ B) - $33 \min (95 \%$ B) - $39 \min (95 \%$ B) - $40 \mathrm{~min}$ $(4 \% \mathrm{~B})-45 \min (4 \% \mathrm{~B})$.

The LC/MS platform was controlled using MassHunter data acquisition software version B.06.00 (Agilent) and the mass spectrometer was operated in positive ionization mode using the following settings: Gas temperature and flow at $150^{\circ} \mathrm{C}$ and $11 \mathrm{~L} / \mathrm{min}$, respectively, iFunnel high and low pressure RF voltages at 110 and $60 \mathrm{~V}$, fragmentor voltage $380 \mathrm{~V}$, cell accelerator voltage $4 \mathrm{~V}$ and capillary voltage $2040 \mathrm{~V}$. Quadrupoles 1 and 2 were set to unit resolution and the collision energy $(\mathrm{CE})$ for each peptide precursor was calculated using equation $\mathrm{CE}=0.036 \mathrm{x}$ $\mathrm{m} / \mathrm{z}-4.8$. 


\section{Reproducibility experiments}

To assess the impact of $\mathrm{HpH}$ reversed-phase fractionation pre-treatment on the reproducibility of our assay, concentrated CM with $0.1 \%$ FCS and cytokine spike-in level of $500 \mathrm{pg} / \mathrm{mL}$ was processed for MS analysis (i.e. reduction, alkylation and digestion) in technical quadruplicates. After digestion, part of each of the four digested replicates was left untreated and frozen at $-80^{\circ} \mathrm{C}$, whereas the remaining amount was exposed to the described fractionation protocol. Lastly, unfractionated and fractionated quadruplicates were analysed via nano-LC/MRMMS injecting $1 \mu \mathrm{g}$ protein per sample on two occasions 13 days apart. Samples were stored at $-80^{\circ} \mathrm{C}$ between the two runs. The methods for sample preparation, fractionation and MRM/MS acquisition for this experiment were the same as previously described.

\section{Data analysis}

Skyline-daily software was used for method development and refinement as well as for subsequent data processing and visualisation. ${ }^{8}$ A retention time predictor was generated based on acquired cytokine peptide results to increase confidence of peptide identification in subsequent samples. Peak picking was manually inspected and adjusted accordingly by comparing retention time and normalised peak area information to the cytokine standard reference runs. The relative ratio of each transition normalised to the total peak area acts as a 'fingerprint' for a compound and is therefore a crucial indicator of a positive identification in complex matrices. In addition, all peaks were manually checked for correct integration, and peak area for each peptide (= sum of all transitions) was exported for further analysis (e.g. calibration curve generation). LOD and LOQ were calculated by the following formulas: $\mathrm{LOD}=3 \sigma / \mathrm{S}$ and $\mathrm{LOQ}=10 \sigma / \mathrm{S}$, where $\sigma=$ the standard deviation of the response based on the calibration curve and $\mathrm{S}=$ the slope of the calibration curve. To quantify the degree of variation for cytokine peptide signals across replicates and multiple MS runs, the coefficient of variation was calculated. The intraday percentage CV was defined for each peptide as the standard deviation between replicates measured within the same day divided by the average peptide signal (normalised peak area) of these replicates times 100. The interday CV (\%) was calculated as follows: standard deviation between replicate measurements on two separate days for each peptide divided by its normalised peak area (average of 4 replicates on 2 days each, $n=8$ ) x 100. All graphs were prepared with GraphPad Prism v7.0 (GraphPad Software, Inc., La Jolla, CA, US). 
Figure S1: Absolute number and percentage of cytokines with detectable peptides showing linearity

(a)

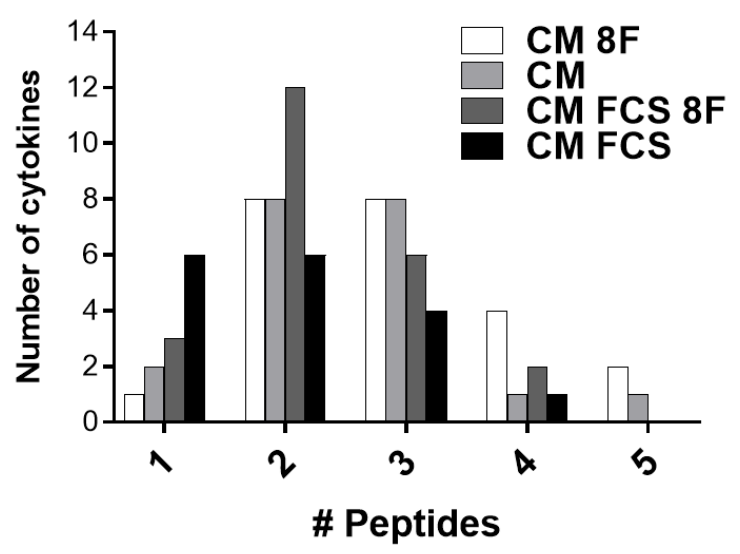

(b)

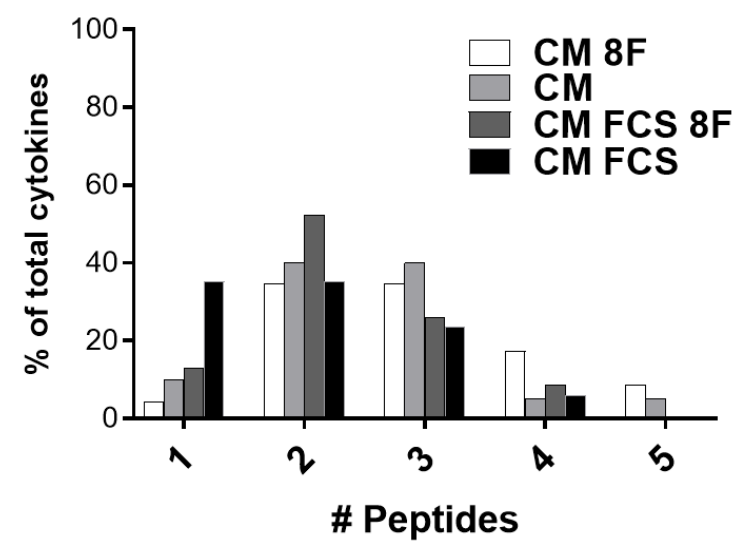

Indicated is the (a) absolute number or (b) percentage of cytokines with detectable peptides which show linearity (= detectable at minimum of three concentration levels) across the four conditions:

Conditioned media (CM) with or without added serum (+/-FCS), and with or without prior fractionation step (+/- 8F). 
Figure S2: Examples of calibration curves and MS signal intensity of internal standard ovalbumin

(a)

\begin{tabular}{|c|l|c|l|l|}
\hline & CM 8F & CM & CM FCS 8F & CM FCS \\
\hline R square & 0.997 & 0.9983 & 0.9763 & 0.9783 \\
\hline
\end{tabular}

\section{IL-18_ISTLSCENK}

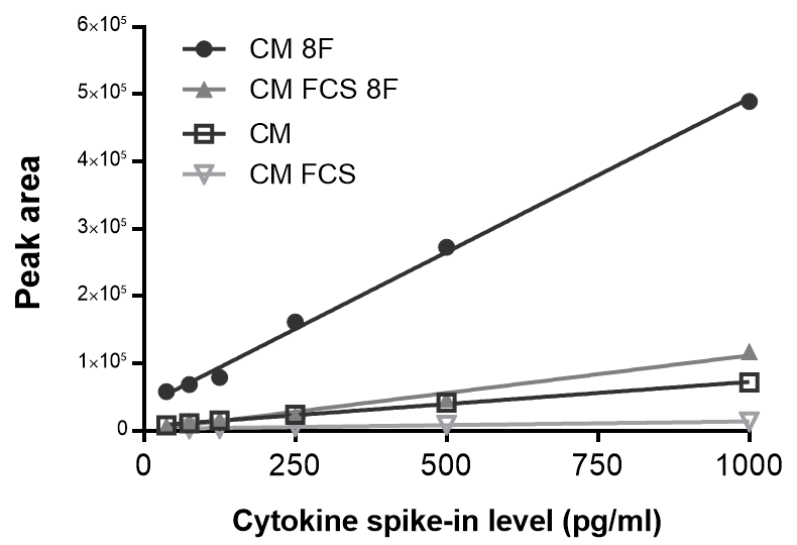

\begin{tabular}{|r|c|c|l|l|}
\hline & CM 8F & CM & CM FCS 8F & CM FCS \\
\hline R square & 0.9974 & 0.9686 & 0.9479 & 0.9999 \\
\hline
\end{tabular}

\section{IL18_SDIIFFQR}

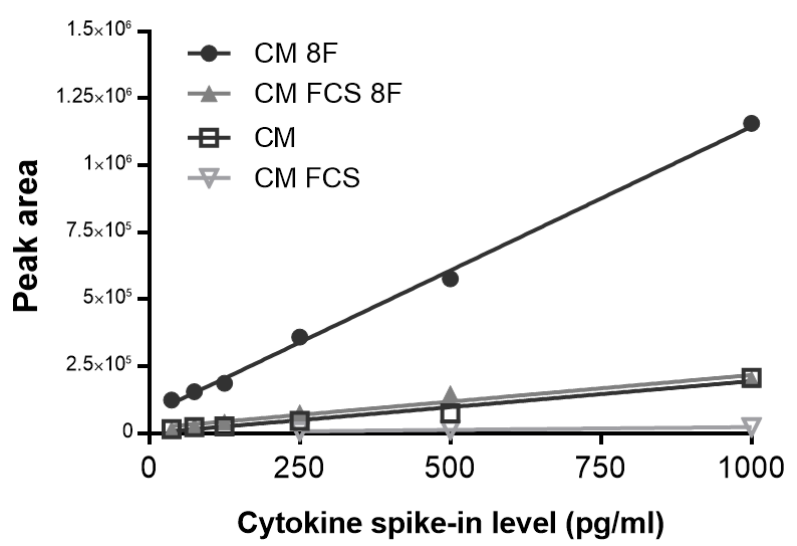

(b)

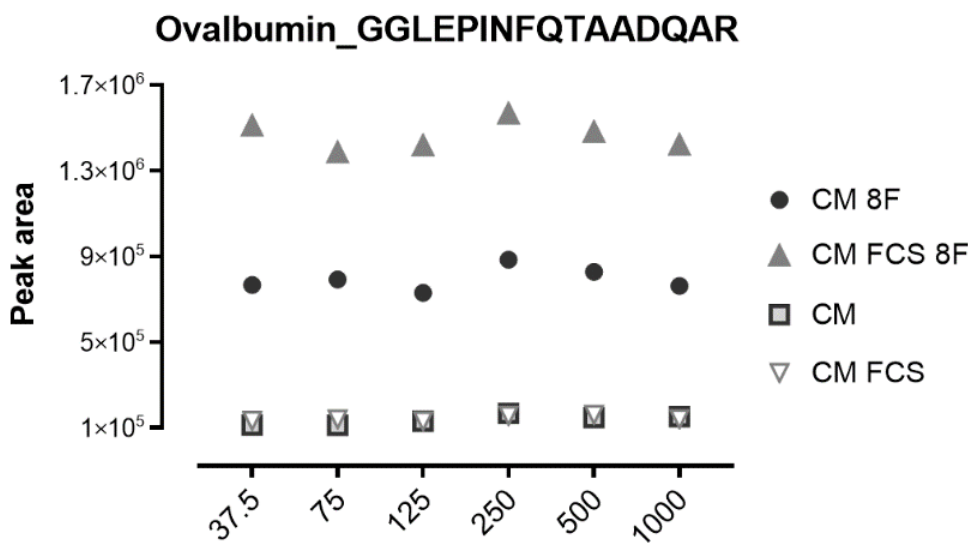

Cytokine spike-in level (pg/ml)

(a) Examples of calibration curves of two peptides belonging to interleukin 18 which were detected at all six calibration levels in CM with or without added serum (+/- FCS), and with or without prior high $\mathrm{pH}$ reversed-phase fractionation $(+/-8 \mathrm{~F})$.

(b) Relatively constant MS signal intensity of internal standard chicken ovalbumin across different conditioned media (CM) samples with multiple cytokine spike-in levels indicates consistent sample preparation and stable mass spectrometer performance. 